\title{
Biomedical Cyber-Physical Systems in the Light of Database as a Service (DBaaS) Paradigm
}

Type of article: Conference abstract

\author{
Maria A. de Jesus ${ }^{1}$, Vania V. Estrela ${ }^{1}$, Ana C. B. Monteiro ${ }^{2}$, Reinaldo P. França ${ }^{2}$, A. Khelassi ${ }^{3}$, Yuzo \\ Iano $^{2}$, Navid Razmjooy ${ }^{4}$, Edwiges G. H. Grata ${ }^{1}$ \\ ${ }^{1}$ State University of Campinas (UNICAMP), Campinas, SP, Brazil \\ ${ }^{2}$ Federal Fluminense University, Rio de Janeiro, Brazil \\ ${ }^{3}$ University of Tlemcen, Tlemcen, Algeria \\ ${ }^{4}$ Department of Electrical Engineering, Tafresh University, Tafresh, Iran \\ Corresponding Author: vania.estrela.phd@ieee.org
}

\begin{abstract}
:
Background: A database (DB) to store indexed information about drug delivery, test, and their temporal behavior is paramount in new Biomedical Cyber-Physical Systems (BCPSs). The term Database as a Service (DBaaS) means that a corporation delivers the hardware, software, and other infrastructure required by companies to operate their databases according to their demands instead of keeping an internal data warehouse.

Methods: BCPSs attributes are presented and discussed. One needs to retrieve detailed knowledge reliably to make adequate healthcare treatment decisions. Furthermore, these DBs store, organize, manipulate, and retrieve the necessary data from an ocean of Big Data (BD) associated processes. There are Search Query Language (SQL), and NoSQL DBs.

Results: This work investigates how to retrieve biomedical-related knowledge reliably to make adequate healthcare treatment decisions. Furthermore, Biomedical DBaaSs store, organize, manipulate, and retrieve the necessary data from an ocean of Big Data (BD) associated processes.

Conclusion: A NoSQL DB allows more flexibility with changes while the BCPSs are running, which allows for queries and data handling according to the context and situation. A DBaaS must be adaptive and permit the DB management within an extensive variety of distinctive sources, modalities, dimensionalities, and data handling according to conventional ways.
\end{abstract}

Keywords: Database as a Service, Data Dimensionality, Healthcare Data, CyberPhysical Systems, Big Data.

\section{Conflict of interest statement}

This article is a conference abstract presented at the International Congress on Health Sciences and Medical Technologies, Tlemcen Algeria 5-7 December 2019, ICHSMT'19.

\section{Authors' biography}

No Biography.

\section{References}

No references 